\title{
A dynamic model to analyse intravenous glucose and insulin tolerance tests performed on dairy cows
}

\author{
Sophie Lemosquet* and Philippe Faverdin \\ Unité Mixte de Recherches sur la Production du Lait, Institut National de la Recherche Agronomique, 35590 Saint Gilles, \\ France
}

(Received 11 October 1999 - Revised 4 April 2001 - Accepted 6 May 2001)

\begin{abstract}
A dynamic model was developed to assess insulin sensitivity and pancreatic response in lactating dairy cows. The model is based on a simultaneous analysis of insulin and glucose intravenous tolerance tests. It comprises five compartments corresponding to insulin in portal-hepatic plasma, and insulin or glucose in both systemic plasma and in interstitial fluid. Insulin secretion rate is a sigmoidal function of glucose in plasma. Insulin is cleared from hepatic plasma and from the interstitial fluid. The glucose entry rate is constant and glucose utilization rate is a sigmoidal function of insulin in the interstitial fluid. Six parameters were estimated: two for insulin secretion rate, two for insulin clearance, one for glucose entry rate and one for glucose utilization rate. After integration of the functions, the model yielded a relative estimate of the quantities of insulin secreted and cleared, as well as the glucose entering and utilized during each test. Using an experimental dataset composed of ten pairs of tolerance tests, the explained variations for plasma insulin and glucose concentrations were 96.0 and $98.3 \%$ and standard errors of estimates were $0.032 \mathrm{nmol} / 1$ and $0.14 \mathrm{mmol} / \mathrm{l}$ respectively. Except in the early stages after injection, residual errors were low. A Jackknife analysis showed that the estimated parameters exhibited low statistical bias. This model simplifies the interpretation of both tests through a simulation based on six common parameters. Compared to a classical analysis of tolerance tests, it may improve the analysis of modifications in the key functions regulating glucose homeostasis in ruminants.
\end{abstract}

Model: Insulin: Dairy cows: Tolerance tests

In comparison with single-stomached mammals, the regulation of glucose metabolism in dairy ruminant animals presents several peculiarities. Due to the fermentation of carbohydrates in the rumen, the intestinal absorption of glucose is limited and the hepatic production of glucose is particularly important. Insulin regulates glucose utilization in extra-mammary peripheral tissues, (including adipose and muscle). In this way, it affects the partitioning of the main nutrients that are precursors for the synthesis of milk constituents between the mammary gland and other tissues. This explains why many authors studying the effects of different physiological and nutritional states on the composition of milk in ruminant animals have analysed the regulation of glucose homeostasis (Sechen et al. 1989; Chilliard \& Ottou, 1995; Lemosquet et al. 1997).

The secretion of insulin interacts with insulin sensitivity in regulating the plasma glucose concentration. The most direct techniques for the in vivo estimation of these two components of glucose homeostasis regulation are difficult and costly to perform on dairy cows from an experimental point of view. These methods involve the use of cows that are catheterized in the hepatic portal vein in order to estimate insulin secretion, and the use of a glucose tracer during euglycaemic hyperinsulinaemic clamps to assess glucose response to insulin (Debras et al. 1989). Intravenous glucose and insulin tolerance tests (injection of a bolus of glucose or insulin) are experimentally easier to perform. However, their interpretation is limited to the analysis of parameters that are calculated from curves of plasma insulin and plasma glucose concentrations. These parameters include peak value, area under the concentration curve and fractional disappearance rate of glucose. The use of mathematical models in biology has improved the treatment of results obtained from different experimental techniques.

Abbreviations: GERO, glucose entry rate; GUM, parameter allowing maximum insulin-stimulated glucose uptake under euglycaemic conditions; GUR, glucose utilization rate; ISM, maximal glucose-stimulated insulin secretion; ISR, insulin secretion rate; KIS, glucose sensitivity parameter of insulin secretion rate.

* Corresponding author: Dr S. Lemosquet, fax +3322348 51 01, email lemosque@st-gilles.rennes.inra.fr 
Among the models for studying the regulation of glucose homeostasis (Cobelli et al. 1982; Castillo et al. 1994; Hovorka \& Jones, 1994), mechanistic models (HOMA, CIGMA or minimal model) have been developed in medicine to allow a more rigorous analysis of results (Pacini \& Bergman, 1986; Turner et al. 1990). These models have simple structures to facilitate the estimation of parameters. For example, the Bergman minimal model, which is used in clinical research, leads to the calculation of four parameters from a glucose tolerance test. Two of the parameters serve to assess the pancreatic response to glucose (first and second phases of secretion), while the other two are used to estimate the insulin sensitivity (Pacini \& Bergman, 1986).

The aim of the present study was to design a dynamic model for glucose and insulin tolerance tests performed on dairy ruminant animals. This model, based simply on measurements of plasma insulin and glucose concentrations, is intended to estimate separately the quantities of secreted and cleared insulin, as well as the total amounts of glucose produced and absorbed or the amount of glucose utilized during the tolerance tests. The insulin tolerance test should improve the evaluation of insulin clearance and facilitate the separate estimates of insulin secretion and clearance during the glucose tolerance test. For this reason, the model was built up from a simultaneous analysis of both tolerance tests.

\section{The model \\ Construction}

The model design was chosen to mimic insulin and glucose distribution in the most important body fluid compartments and to simulate the key regulatory functions of glucose homeostasis. Insulin (Fig. 1) diffuses within three compartments, which represent the quantity of insulin in systemic plasma $\left(I_{1}\right.$, nmol $)$, portal-hepatic plasma $\left(I_{3}, \mathrm{nmol}\right)$ and interstitial fluid $\left(I_{2}\right.$, nmol) in accordance with the models proposed for humans subjects by Ferrannini \& Cobelli (1987). Insulin is injected $\left(I_{I N J}, \mathrm{nmol}\right)$ in the systemic plasma compartment $I_{1}$ (Fig. 1) during the insulin tolerance test. It is cleared from the interstitial fluid compartment $I_{2}$ with a fractional disappearance rate $K I_{02}\left(\mathrm{~min}^{-1}\right)$ or from the portal-hepatic plasma compartment of insulin $I_{3}$ with a fractional disappearance rate $K I_{03}\left(\mathrm{~min}^{-1}\right)$, as proposed in the model of Ferrannini \& Cobelli (1987). Insulin is secreted in the portal-hepatic plasma compartment of insulin $I_{3}$. The insulin secretion rate function (ISR) increases similar to a sigmoidal curve as a function of glucose concentration ( $g l y c, \mathrm{mmol} / \mathrm{l}$ ). It corresponds to the function observed for single-stomached mammals in vivo as well as in vitro (Ashcroft, 1981).

There are numerous similarities between modelling procedures used for glucose and insulin. Glucose diffuses in only two compartments (Fig. 1), where the systemic plasma is represented by $G_{1}(\mathrm{mmol})$ and the interstitial fluid by $G_{2}(\mathrm{mmol})$. A specific compartment for the diffusion of glucose in the portal-hepatic system was not necessary since the uptake of glucose by the liver is negligible in ruminants (Hocquette et al. 1996). The plasma compartment

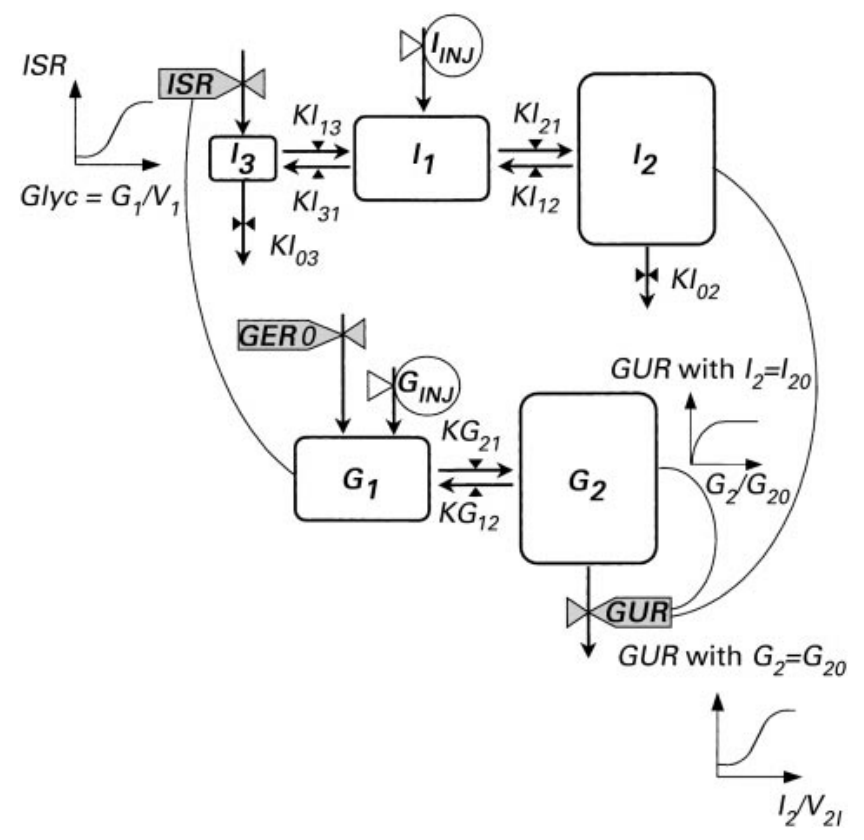

Fig. 1. Compartments and parameters of the model. For further details of the model, see p. $360 . I_{1}, I_{2}, I_{3}$, quantities of insulin in compartments, comparable with the systemic plasma, the interstitial fluid and the hepatic plasma respectively; $G_{1}$ and $G_{2}$, quantities of glucose in compartments comparable with the systemic plasma and the interstitial fluid respectively; $K I_{12}, K I_{21}, K I_{13}, K I_{31}, K G_{12}, K G_{21}$, coefficients of transfer rates between compartments; $I_{I N J}$, quantity of insulin injected; ISR, insulin secretion rate function; KI $I_{02}$ and $K I_{03}$, insulin fractional disappearance rates; $G_{I N J}$, quantity of glucose injected; GER0, glucose entry rate function; GUR, glucose utilization rate function; Glyc, plasma glucose concentrations.

$G_{1}$ receives the glucose injected $\left(G_{I N J}, \mathrm{mmol}\right)$ during the glucose tolerance test. It also receives the hepatic glucose output represented by the glucose entry rate function (GER0). This function is assumed to be constant in the model but its value is always estimated during the simulations. Glucose from the interstitial fluid compartment $G_{2}$ can be utilized by tissue. The glucose utilization rate function $(G U R)$ depends on both the insulin and glucose concentrations (Fig. 1). In ruminants, as in singlestomached mammals, the total glucose utilization is higher under hyperglycaemic hyperinsulinaemic clamps than under euglycaemic hyperinsulinaemic clamps (Bergman et al. 1989). Under euglycaemic conditions, $G U R$ is a sigmoidal function (Debras et al. 1989) of the quantity of insulin $I_{2}$ in the interstitial fluid compartment (Fig. 1). Under euinsulinaemic conditions, GUR increases curvilinearly in response to increasing glucose in compartment $G_{2}$ (Fig. 1) as in the model proposed by DeFronzo \& Ferrannini (1982).

\section{Model parameterization}

The volumes of the compartments are $V_{1}$ (litres), $V_{2 \mathrm{I}}$ (litres) and $V_{3 \mathrm{I}}$ (litres), for $I_{1}, I_{2}$, and $I_{3}$ respectively. For $G_{1}$ and $G_{2}$, these volumes correspond to $V_{1}$ (litres) and $V_{2 \mathrm{G}}$ (litres) respectively. In the basal state, the concentrations of plasma insulin and plasma glucose, and the five quantities of insulin 
and glucose, are denoted as insul0, glyc0, $I_{10}, I_{20}, I_{30}, G_{10}$ and $G_{20}$ respectively. The parameters $K I_{m n}$ and $K G_{m n}$ represent constant fractional transfer rates from compartment $I_{n}$ to compartment $I_{m}$ and from compartment $G_{n}$ to compartment $G_{m}$ respectively. The equations for the insulin and glucose submodels can then be written as follows:

$$
\begin{gathered}
\frac{d I_{1}}{d t}=K I_{13} \times I_{3}+K I_{12} \times I_{2}-\left(K I_{21}+K I_{31}\right) \times I_{1}, \\
\frac{d I_{2}}{d t}=K I_{21} \times I_{1}-\left(K I_{12}+K I_{02}\right) \times I_{2}, \\
\frac{d I_{3}}{d t}=I S R+K I_{31} \times I_{1}-\left(K I_{13}+K I_{03}\right) \times I_{3}, \\
\frac{d G_{1}}{d t}=G E R 0+K G_{12} \times G_{2}-K G_{21} \times G_{1},
\end{gathered}
$$

and

$$
\frac{d G_{2}}{d t}=K G_{21} \times G_{1}-K G_{12} \times G_{2}-G U R .
$$

We used a logistic equation to describe the sigmoidal curve of ISR (Sturis et al. 1991):

$$
I S R(\mathrm{nmol} / \mathrm{min})=I S N G D+\frac{I S M}{\left.1+I S 1 \times \mathrm{e}^{(K I S \times(g l y c 0-g l y c)}\right)},
$$

where ISNGD corresponds to the non-glucose-dependent insulin secretion, ISM corresponds to the maximal glucosestimulated insulin secretion and KIS expresses the pancreatic sensitivity to glucose. The logistic curve is skew-symmetric, with an inflection point at:

$$
x=\operatorname{glyc} 0+\frac{\ln (I S 1)}{K I S}
$$

and at:

$$
y=\frac{I S M}{2} .
$$

We also decided to describe the $G U R$ function under euglycaemic conditions $\left(G_{2}=G_{20}\right)$ using a 'logistic equation' and under euinsulinaemic conditions $\left(I_{2}=I_{20}\right)$ using an exponential equation. The GUR function was obtained by directly multiplying the equations describing the dependence on glucose and insulin, in accordance with most models of glucose utilization in single-stomached mammals (Sturis et al. 1991). With such a function, the utilization of glucose is nil when the quantity of glucose in compartment $G_{2}$ is nil, which prevents the glucose compartments from taking on negative values:

$$
\begin{aligned}
& \text { GUR }(\mathrm{mmol} / \mathrm{min})= \\
& \left(G U N D+\frac{G U M}{1+G U 1 \times \mathrm{e}^{\left(K G U I \times \frac{\left(I_{20}-I_{2}\right)}{V_{2 I}}\right)}}\right) \\
& \times\left(1-\mathrm{e}^{\left(-K G U G \times \frac{G_{2}}{G_{20}}\right)}\right),
\end{aligned}
$$

Under euglycaemic conditions, $G U 1$ sets the position of inflection of the curve on the $x$-axis and $K G U I$ expresses the insulin sensitivity to glucose. The maximal insulinstimulated glucose utilization is represented by $G U M \times(1-$ $\left.\mathrm{e}^{(-K G U G)}\right)$. Under euinsulinaemic conditions, $K G U G$ represents the GUR sensitivity to glucose and, as in the model of Sturis et al. (1991), the non-insulin dependent glucose utilization corresponds to $G U N D \times\left(1-\mathrm{e}^{\left(-K G U G \times \frac{G_{2}}{G_{20}}\right)}\right)$.

\section{Model constraints}

The complexity of the model was considerable: it included seven equations and twenty-three parameters. Several constraints were adopted to limit the number of parameters estimated to six in the final version of the model. This model is a compromise between a physiologically justifiable description and a representation whose parameters can be estimated from experimental data. The six parameters estimated were chosen from the twelve that describe modifications in the glucose homeostasis functions (i.e. secretion and clearance of insulin, entry and utilization of glucose).

We decided first of all to fix the transfer rates between compartments $\left(K I_{21}=0.12, K G_{21}=0 \cdot 25, K I_{31}=0.35\right)$ and all the parameter values in order to calculate the compartment volumes. The volume $V_{1}$ in the plasma compartments of insulin and glucose $\left(I_{1}\right.$ and $\left.G_{1}\right)$ increased with the body weight $(B W)$ and the milk yield (MILK) according to Pfeffer et al. (1965) and Bergman et al. (1989):

$$
V_{1}(\text { litres })=0.36 \times M I L K+0.0315 \times B W .
$$

This regression function was calculated using our experimental data ( $n$ 16, $R^{2}$ 0.986). The volumes of insulin $V_{2 I}$ and glucose $\mathrm{V}_{2 G}$ in interstitial fluid compartments $\left(I_{2}, G_{2}\right)$ were fixed at 14 and $11 \%$ BW respectively. The portal-hepatic fluid volume of the insulin compartment $\left(I_{3}\right)$ was fixed at $18 \% V_{1}$ according to Campra \& Reynolds (1988). For a cow producing $30 \mathrm{~kg} \mathrm{milk/d}$, the total body fluid diffusion of insulin and glucose represents 19.8 and $16 \%$ BW respectively. These values are in agreement with Pfeffer et al. (1965) and Bergman et al. (1989).

Second, the parameter GUND was determined after calculating the non-insulin dependent glucose utilisation under basal conditions (when $G_{2}=G_{20}$ ). We built up an equation using milk yield data to calculate this fraction of glucose utilization. In dairy ruminant animals, mammary gland glucose uptake is not mediated by insulin and is 
quantitatively very important (Debras et al. 1989). About $80 \%$ glucose taken up by the mammary gland is secreted as lactose, which has a concentration of about $50 \mathrm{~g} / 1 \mathrm{milk}$ (Annison et al. 1974). The other fraction of non-insulin dependent utilization in other organ tissues was fixed at $300 \mathrm{mg} / \mathrm{min}$ using data of glucose brain uptake in sheep (Pell \& Bergman, 1983 quoted by Hocquette \& Balange, 1996). It represents approximately $10 \%$ total glucose utilization in a cow producing $30 \mathrm{~kg}$ milk/d. The parameter GUND (mmol/l) was then calculated using:

$$
\begin{aligned}
& G U N D \times\left(1-\mathrm{e}^{-K G U G}\right)= \\
& \quad\left(300+\frac{\left(M I L K \times 0.05 \times 10^{6}\right)}{0.8 \times 24 \times 60}\right) \times \frac{1}{180} .
\end{aligned}
$$

Finally, we assumed that the basal state corresponds to an initial equilibrium state for insulin and glucose. As described in Hovorka et al. (1993), the net fluxes of insulin and glucose between the different compartments are assumed to be nil if the concentration gradient is zero, thus giving:

$$
K I_{12}=K I_{21} \times \frac{V_{1}}{V_{2 I}} .
$$

Similar relations were used between $K I_{13}$ and $K I_{31}$ or between $K G_{12}$ and $K G_{21}$. In addition:

$$
\frac{d I_{1}}{d t}=0, \frac{d I_{2}}{d t}=0, \quad \frac{d I_{3}}{d t}=0, \quad \frac{d G_{1}}{d t}=0 \quad \text { and } \quad \frac{d G_{2}}{d t}=0 .
$$

The parameters $I_{20}, I_{30}, G_{20}$ and the insulin secretion rate under basal conditions ISR0 can then be calculated by solving equations $1,2,4,5$ and 3 . The value of $I S 1$, can then be obtained using equation 3 . At basal state, the glucose entry rate function $(G E R 0)$ and the glucose utilization rate function $(G U R 0)$ are also equilibrated. The value of $G U 1$ can then be derived using equation 7 .

After imposing these constraints, there are nine remaining parameters to estimate (ISM, KIS, $K I_{02}, K_{03}, G E R 0, G U M$, ISNGD, KGUI, KGUG), from which six were chosen after assessing the quality of the simulation (see p. 362).

\section{Application}

The input variables in the model (i.e. experimental data required) are the animal's body weight $(B W, \mathrm{~kg})$, its milk yield $(M I L K, \mathrm{~kg} / \mathrm{d})$, the quantities of glucose $\left(G_{I N J}, \mathrm{mmol}\right)$ and insulin $\left(I_{I N J}, \mathrm{nmol}\right)$ injected during glucose and insulin tolerance tests, the plasma glucose concentration ( $g l y c$, $\mathrm{mmol} / \mathrm{l}$ ) and the plasma insulin concentration (insul, $\mathrm{nmol} / \mathrm{l})$, the time $(t, \mathrm{~min})$ of injection and sampling.

\section{Experimental data}

The experimental datasets from trial 2 of the study published by Lemosquet et al. (1997) were used to develop the present model. Four dairy cows were fed a diet based on dehydrated whole plant maize and received a continuous infusion of glucose into the duodenum $(1500 \mathrm{~g} / \mathrm{d})$ or water (control infusion) according to a switch-back design with periods of 4 weeks. A glucose tolerance test and an insulin tolerance test were performed on the same cow during the last week of each period. Cows weighed $635 \mathrm{~kg}$ and yielded $32.7 \mathrm{~kg}$ milk/d under the control infusion and $31.6 \mathrm{~kg}$ milk/d under duodenal glucose infusion. The model was then developed using eight sets of individual data (four cows receiving two treatments) and two sets of mean data corresponding to the means of the experimental treatments (control $v$. duodenal glucose infusion). In the model, the injections of glucose and insulin were simulated as being instantaneous at time $t=0.5 \mathrm{~min}$. A total of twenty-six data points of insulin and glucose concentration were used to carry out each simulation. The mean glucose and insulin concentrations taken before the injection ( $g l y c 0$ and insul0 respectively) were introduced in the model in order to define the basal equilibrium state at time $t=0 \mathrm{~min}$. The concentrations of glucose and insulin at $t<5$ min were not taken into account since the model does not allow us to describe the diffusion of products into the first compartments immediately after injection.

\section{Data simulation}

The model program is written in ACSL (Mitchell \& Gauthier, Inc., Concord, MA, USA). The optimizations were performed using the SIMUSOLV software (Dow Chemical Company, Midland, MI, USA). The system of differential equations was solved through numerical integration using Gear's backward difference formula in the Simusolv program (Steiner et al. 1990). The integration step-size was varied but did not exceed the interval between observations ( $3 \mathrm{~min})$. Parameters were estimated by maximization of the log likelihood function (Bard's likelihood standard reduced model; Steiner et al. 1990) using insulinaemia and glycaemia as time-dependent variables. The simulation with Simusolv included a model for the asymptotic standard errors (ASE) of the predicted value for a variable $(f)$ :

$$
\operatorname{ASE}^{2}=\alpha^{2} \times f^{\gamma}
$$

where $\alpha$ is a proportionality factor and $\gamma$ is called the heteroscedasticity parameter which is also estimated during the simulation (Steiner et al. 1990).

The simulations were performed with the same set of initial parameter values $\left(K I_{02} 0.07 \mathrm{~min}^{-1}, K I_{03} 0.65 \mathrm{~min}^{-1}\right.$, ISNGD $0.314 \mathrm{nmol} / \mathrm{min}$, KIS 1.08 , ISM $6.10 \mathrm{nmol} / \mathrm{min}$, GER0 $16.67 \mathrm{mmol} / \mathrm{min}, K G U I$ 28.67, KGUG 1.8, GUM $25 \mathrm{mmol} / \mathrm{min})$. The final model uses a simulation with six parameters to be estimated $\left(K I_{02}, K I_{03}, K I S, I S M, G E R 0\right.$ and $G U M)$. At the end of each simulation, the total amounts of insulin secreted $\left(I S_{T}\right)$ during each test were calculated by integration of the different functions over $80 \mathrm{~min}$ (i.e. the total duration of the test plus $5 \mathrm{~min}$ ). The same applies for the total amount of insulin cleared $\left(I C L 2_{T}\right.$ for $I_{2}, I C L 3_{T}$ for $\left.I_{3}, I C L_{T}=I C L 2_{T}+I C L 3_{T}\right)$, as well as the total amounts of glucose entering $\left(G E_{T}\right)$ and glucose utilized $\left(G U_{T}\right)$.

\section{Method of analysis and validation of the model}

The purpose of the different simulations was to study the quality of the model mainly by analysis of the residuals (difference between observed data and estimated data). 
To study the variation of the model parameters during optimization, a procedure known as the Jackknife method and a sensitivity analysis were applied on the set of mean data obtained with the control treatment. The Jackknife method is effective in obtaining, at the same time, nonbiased estimates of the parameters and standard errors of the quantities of secreted insulin, cleared insulin $\left(I S_{T}\right.$ and $\left.I C L_{T}\right)$ and of glucose entering and utilized $\left(G E_{T}\right.$ and $\left.G U_{T}\right)$ during each tolerance test. This method is also performed to study the contribution of sampling times in determining parameter values. The procedure is based on a resampling of data (Simonoff \& Tsai, 1986). Twenty-six simulations were carried out, and from each simulation the data corresponding to a sampling time in one of the two tests were removed, leading to twenty-six sets of pseudo-values. A standard Jackknife estimate is defined as the mean value of the twenty-six corresponding pseudo-values. The sensitivities of $I S_{T}, I C L 2_{T}$ and $I C L 3_{T}, G U_{T}$ and $G E_{T}$ were tested in response to variations of $+20 \%$, the initial value of one fixed parameter. For example, the sensitivity of $I S_{T}$ to the fixed parameter $V_{1}$ will be equal to 1 if $I S_{T}$ increased $20 \%$ in response to a $20 \%$ increase of $V_{1}$.

Datasets of glucose and insulin tolerance tests from Chilliard \& Ottou (1995) and from Sechen et al. (1989) were used to validate the model. The datasets corresponded to mean data from each experimental treatment. The simulations were then performed under similar conditions to our experimental data. The parameter $V_{2 G}$ was the only value modified in the set of initial parameters $(0 \cdot 14$ instead of $0.11 \% \mathrm{BW}$ ).

\section{Results of simulations}

In the simulation carried out in this study, six parameters were chosen (ISM, KIS, KI $I_{02}, K I_{03}, G E R 0$ and GUM)

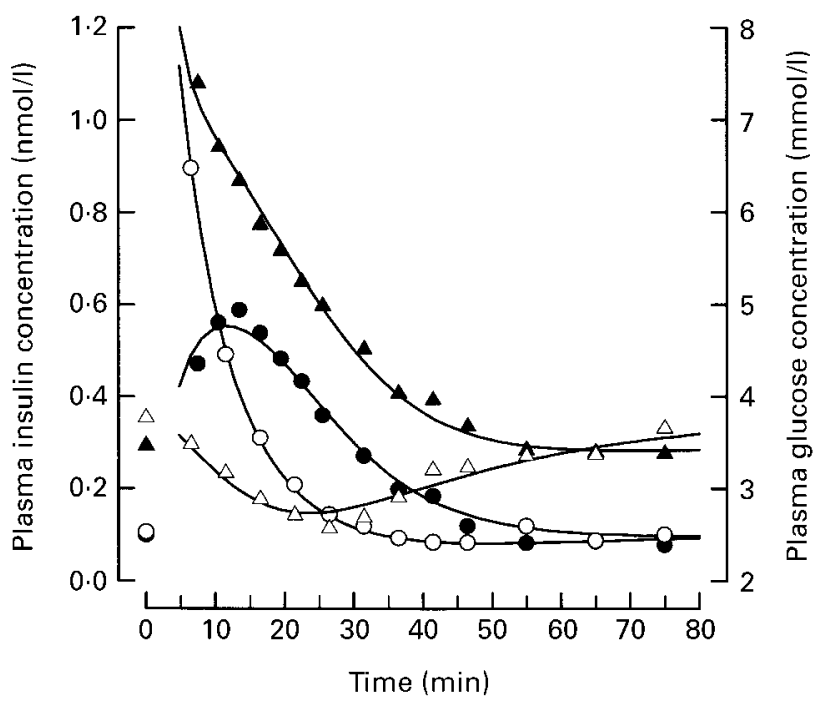

Fig. 2. An example of model fitting for plasma insulin $(\bullet, \bigcirc)$ and plasma glucose $(\boldsymbol{\Lambda}, \Delta)$ concentrations during a glucose tolerance test $(\boldsymbol{\square}, \boldsymbol{\Delta})$ and an insulin tolerance test $(O, \Delta)$ (based on mean values of control treatment). For details of the model and procedures see pp. 360-362. from the nine that could be estimated (see model constraints, p. 361). This was done both to limit high correlations between parameters and to obtain the best fit of plasma glucose and insulin concentrations (Fig. 2). We found that the model converges rapidly when the number of estimated parameters is between five and nine, but the number of highly correlated parameters increases when there are more than eight estimated parameters and their estimations become imprecise. Nevertheless, in the present simulation KIS $v$. ISM are highly correlated $(-0.83)$ and GUM v. GER0 as $K I_{02}$ v. $K I_{03}$ yield correlation coefficients higher than 0.5 (0.69 and 0.68 respectively). On eight datasets (four cows receiving the control treatment and the duodenal glucose infusion treatment), the variation explained was on average $96.0 \%$ for the plasma insulin concentration and $98.3 \%$ for the plasma glucose concentration, while the respective standard errors of the estimates were $0.032 \mathrm{nmol} / 1$ and $0.14 \mathrm{mmol} / \mathrm{l}$. The analysis of the residuals (Fig. 3) indicates that the model fails to fit the insulin and the glucose curves at the first sampling time of the glucose tolerance test $(t=7.5 \mathrm{~min})$ when the concentrations are high. Indeed, the model tends to overestimate the insulin concentration at the first sampling time and to underestimate the high concentrations of glucose. In addition, it tends to overestimate the minimum values of glucose in the insulin tolerance tests.

The results of parameter estimation are presented in Table 1. The duodenal glucose infusion significantly increased both GER0, the glucose entry rate, and GUM, the parameter conditioning the maximum of glucose utilization, and decreased $K I_{03}$, the hepatic insulin clearance parameter. The sum of the quantities of insulin injected and secreted $\left(I S_{T}+I_{I N J}\right)$ and the sum of the quantities of insulin cleared $\left(I C L_{T}\right)$ reach equilibrium over the duration of each tolerance test (Table 1). The same applies for the sum of glucose entering $\left(G E_{T}+G_{I N J}\right)$ and utilized $\left(G U_{T}\right)$. Since the glucose entry rate function GERO is constant, it constrains the total quantity of glucose utilized $\left(G U_{T}\right)$ during the tolerance tests.

\section{Results of internal validations}

For the six parameters estimated, as well as for the integrated functions $\left(I S_{T}, I C L_{T}, G E_{T}, G U_{T}\right)$, the means calculated for each treatment (four cows per treatment) yield values close to estimates obtained from the simulation of two datasets. These correspond to mean data for each experimental treatment, despite the non-linearity of the model. Only ISM and KIS show slight differences between the two methods of calculation (means of four datasets $v$. dataset of mean values for control infusion, ISM $5.88 \mathrm{nmol} / \mathrm{min}$ in Table $1 v .7 .72 \mathrm{nmol} / \mathrm{min}$ in Table 2; and for duodenal glucose infusion, KIS $1.00 v .1 \cdot 37$ ). Internal validation (including Jackknife method and sensitivity analysis) was then performed on the dataset corresponding to the mean values for the control treatment.

The simultaneous analysis of both tolerance tests greatly improved the estimation of the six parameters. Table 2 reports the means and the asymptotic standard errors (ASE) obtained when the simulation was performed using data 
(a)

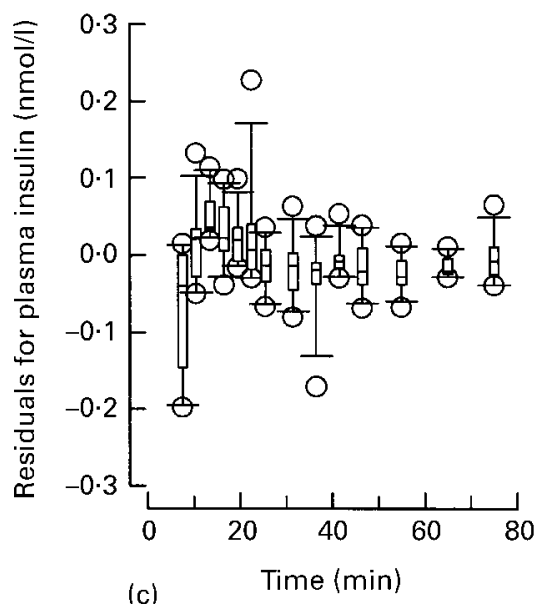

(c)

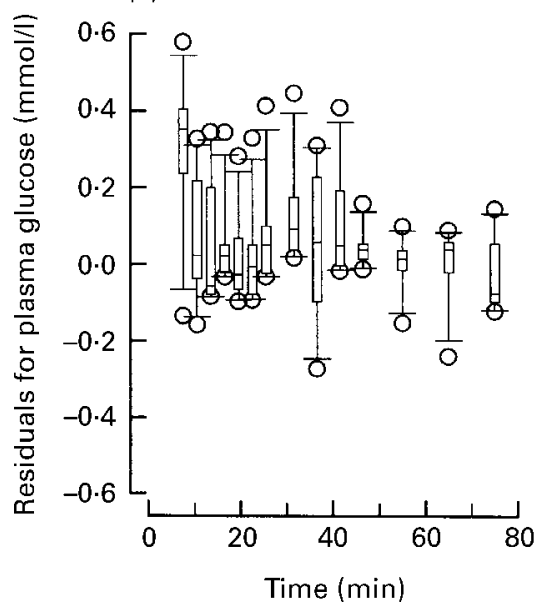

(b)

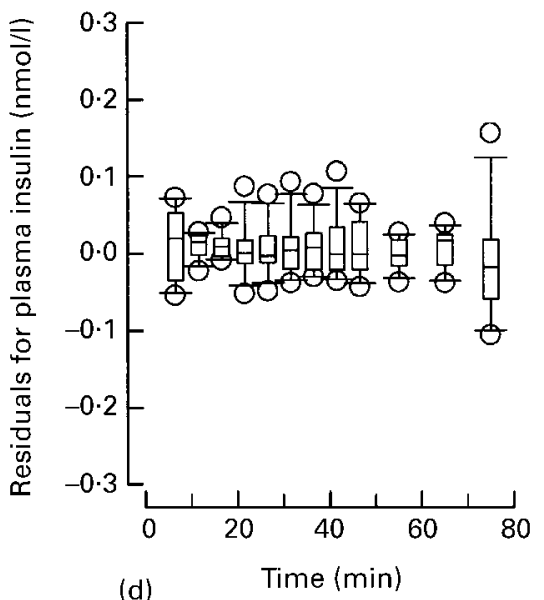

(d)

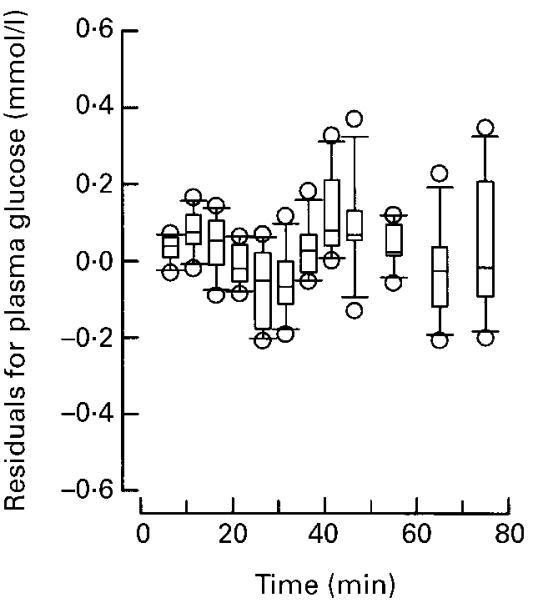

Fig. 3. Box plots graph representing statistical residuals for plasma insulin (a),(b) and glucose (c),(d) concentrations. (a),(c), Glucose tolerance test; (b),(d), insulin tolerance test. For details of the model and procedures, see pp. 360-362. Residuals were calculated as observed-predicted data. The box boundary closest to zero indicates the 25th percentile, while the line within the box marks the median. The box boundary farthest from zero indicates the 75th percentile. Vertical bars above and below the box indicate the 90th and 10th percentiles and circles $(O)$ indicate outlying points.

from both tolerance tests. The ASE greatly increased when the simulation was carried out using data from the glucose tolerance test alone. The mean values are: ISM 7.87 (ASE 4.51) $\mathrm{nmol} / \mathrm{min}$, KIS 0.97 (ASE 0.08), $K I_{03} 0.86$ (ASE 1.04) $\min ^{-1}, K I_{02}$ 0.16 (ASE 0.02) $\min ^{-1}$, GER0 19.6 (ASE 6.0), GUM 21.4 (ASE $4 \cdot 3$ ) $\mathrm{mmol} / \mathrm{min}$. This was also the case for the insulin tolerance test. The mean values are: ISM 5.77 (ASE 51) $\mathrm{nmol} / \mathrm{min}$, KIS 0.72 (ASE 0.77 ) $\mathrm{mmol} / \mathrm{min}, K I_{03}$ 0.47 (ASE 0.020$) \mathrm{min}^{-1}, K I_{02} 0.157$ (ASE 0.03) $\mathrm{min}^{-1}$, GER0 17.5 (ASE 1.4) $\mathrm{mmol} / \mathrm{min}$, GUM 23.5 (ASE 2.9) $\mathrm{mmol} / \mathrm{min}$. The insulin tolerance test greatly improved the precision of estimation of both hormone clearance $\left(K I_{03}\right)$ and the glucose entry rate $(G E R 0)$. The estimations of insulin secretion parameters (ISM, KIS) were only estimated precisely in the glucose tolerance test.

When data from both tolerance tests were used, the nonbiased standard errors of the Jackknife estimates ranged from one to two times the asymptotic standard errors (Table $2)$. The $\mathrm{CV}$ for the parameters obtained with the Jackknife method (Table 2) ranged from 3.6 to $22.4 \%$ : GER0 3.6, KIS
6.6, GUM 6.8, $K I_{03} 7 \cdot 3, K I_{02} 9 \cdot 1$ and $I S M 22.4 \%$. The nonbiased standard errors for the integrated functions $\left(I S_{T}\right.$ and $I C L_{T}, G E_{T}$ and $G U_{T}$ ) calculated by this method were low (Table 2). Their CV ranged from $1 \cdot 2$ to $4 \cdot 2 \%$.

Two examples of the effect of sampling time on the determination of parameter values and integrated function are presented in Fig. 4. Two particular sampling intervals had an important influence on parameter estimations. These sampling intervals correspond to the early stage of the glucose tolerance test $(7 \cdot 5-15 \cdot 5 \mathrm{~min})$, when the insulin and glucose concentrations are maximal for the six estimated parameters, and also when glucose concentrations are minimal (21.5-31.5 $\mathrm{min})$ during the insulin tolerance test for $K I_{02}, G E R 0$ and $G U M$. The values taken by the integrated functions $\left(I S_{T}, I C L_{T}, G E R 0, G U_{T}\right)$ depend on both of these sampling times.

The quantities of insulin that are secreted $\left(I S_{T}\right)$ or cleared $\left(I C L_{T}\right)$ are very sensitive to any increase in the initial values of the parameters, in particular for $I S_{T}$ and $I C L_{T}$ when calculated for the glucose tolerance test. These two 
Table 1. Means of parameter estimates obtained when the simulations were performed on eight datasets corresponding to four cows receiving duodenal infusions of water (control) or glucose $(1500 \mathrm{~g} / \mathrm{d})$ according to a switch-back design*

\begin{tabular}{|c|c|c|c|c|}
\hline & $\begin{array}{c}\text { Control } \\
\text { infusion }(n 4)\end{array}$ & $\begin{array}{l}\text { Duodenal glucose } \\
\text { infusion }(n 4)\end{array}$ & sD† & Statistical significance: $P \ddagger$ \\
\hline ISM(nmol/min) & $7 \cdot 7$ & $8 \cdot 8$ & $1 \cdot 32$ & 0.4 \\
\hline KIS & 0.98 & 1.37 & 0.297 & 0.2 \\
\hline$K I_{02}\left(\min ^{-1}\right)$ & 0.15 & $0 \cdot 13$ & 0.037 & 0.4 \\
\hline$K I_{03}\left(\min ^{-1}\right)$ & 0.50 & 0.27 & 0.062 & 0.03 \\
\hline GERO (mmol/min) & $16 \cdot 7$ & $21 \cdot 0$ & $1 \cdot 11$ & 0.03 \\
\hline GUM (mmol/min) & $22 \cdot 1$ & $24 \cdot 1$ & 0.58 & 0.04 \\
\hline \multicolumn{5}{|l|}{ Glucose tolerance test } \\
\hline Glucose injected (mmol) & 378 & 378 & & \\
\hline$I S_{T}(\mathrm{nmol})$ & 132 & 134 & $42 \cdot 6$ & 0.9 \\
\hline$I C L_{T}(\mathrm{nmol})$ & 132 & 134 & $42 \cdot 9$ & 0.9 \\
\hline$G E_{T}(\mathrm{mmol})$ & 1335 & 1681 & 88.9 & 0.03 \\
\hline $\mathrm{GU}_{T}(\mathrm{mmol})$ & 1717 & 2075 & $90 \cdot 1$ & 0.03 \\
\hline \multicolumn{5}{|l|}{ Insulin tolerance test } \\
\hline Insulin injected (nmol) & 70 & 70 & & \\
\hline$I_{T}(\mathrm{nmol})$ & 44 & 46 & $12 \cdot 1$ & 0.9 \\
\hline$I C L_{T}(\mathrm{nmol})$ & 115 & 116 & $12 \cdot 2$ & 0.9 \\
\hline$G E_{T}(\mathrm{mmol})$ & 1335 & 1681 & 88.9 & 0.03 \\
\hline$G U_{T}(\mathrm{mmol})$ & 1351 & 1691 & $82 \cdot 3$ & 0.02 \\
\hline
\end{tabular}

$I S M$, maximal glucose-stimulated insulin secretion; $K I S$, glucose sensitivity parameter of insulin secretion rate function; $K I_{02}$ and $\mathrm{KI}_{03}$, insulin fractional disappearance rates for compartments 2 and 3; GERO, glucose entry rate function; GUM, parameter allowing maximal insulin-stimulated glucose utilization under euglycaemic conditions; $I S_{T}$, total amount of insulin secreted during a tolerance test; $I C L_{T}$, total amount of insulin cleared; $G E_{T}$, total amount of glucose entering; $G U_{T}$, total amount of glucose utilized.

${ }^{*}$ For details of procedures, see pp. 360-362.

† Residual standard deviation of the ANOVA, (see Lemosquet et al. 1997).

$\ddagger$ Effects of treatments.

Table 2. Comparison of parameter estimates and asymptotic standard errors (ASE) to non-biased parameter estimates and corresponding non-biased standard errors (NBSE) obtained by the Jackknife method (on the dataset corresponding to the means for the control treatment)*

\begin{tabular}{|c|c|c|c|c|}
\hline & \multicolumn{2}{|c|}{ Asymptotic } & \multicolumn{2}{|c|}{ Jackknife method $\dagger$} \\
\hline & Estimates & ASE & Estimates & NBSE \\
\hline ISM (nmol/min) & 5.9 & 0.37 & $5 \cdot 3$ & $1 \cdot 18$ \\
\hline$K I S$ & 0.99 & 0.034 & 1.02 & 0.069 \\
\hline$K I_{02}\left(\min ^{-1}\right)$ & $0 \cdot 18$ & 0.017 & 0.22 & 0.020 \\
\hline$K I_{03}\left(\min ^{-1}\right)$ & 0.44 & 0.032 & 0.42 & 0.031 \\
\hline GERO $(\mathrm{mmol} / \mathrm{min})$ & 15.9 & 0.46 & $15 \cdot 6$ & 0.57 \\
\hline GUM (mmol/min) & $20 \cdot 5$ & 0.94 & $22 \cdot 1$ & 1.50 \\
\hline \multicolumn{5}{|c|}{ Glucose tolerance test } \\
\hline$I S_{T}(\mathrm{nmol})$ & 131 & - & 132 & $5 \cdot 6$ \\
\hline$I C L_{T}(\mathrm{nmol})$ & 131 & - & 132 & $5 \cdot 6$ \\
\hline$G E_{T}(\mathrm{mmol})$ & 1269 & - & 1250 & $45 \cdot 3$ \\
\hline$G U_{T}(\mathrm{mmol})$ & 1651 & - & 1627 & $45 \cdot 0$ \\
\hline \multicolumn{5}{|c|}{ Insulin tolerance test } \\
\hline$I S_{T}(\mathrm{nmol})$ & 43 & - & 43 & 1.4 \\
\hline$I C L_{T}(\mathrm{nmol})$ & 113 & - & 113 & 1.4 \\
\hline$G E_{T}(\mathrm{mmol})$ & 1269 & - & 1250 & $45 \cdot 3$ \\
\hline$G U_{T}(\mathrm{mmol})$ & 1285 & - & 1265 & $42 \cdot 8$ \\
\hline
\end{tabular}

$I S M$, maximal glucose-stimulated insulin secretion; KIS, glucose sensitivity parameter of insulin secretion rate function; $\mathrm{KI}_{02}$ and $K \mathrm{I}_{03}$, insulin fractional disappearance rates for compartments 2 and 3; GERO, glucose entry rate function; GUM, parameter allowing maximal insulin-stimulated glucose utilization under euglycaemic conditions; $I_{T}$, total amount of insulin secreted during a tolerance test; $I C L_{T}$, total amount of insulin cleared; $G E_{T}$, total amount of glucose entering; $G U_{T}$, total amount of glucose utilized.

* For details of procedures, see pp. $360-363$.

†The Jackknife method is effective in obtaining non-biased estimates of the parameters and standard errors of the quantities $I S_{T}, I C L_{T}, G E_{T}, G U_{T}$ simultaneously. quantities are very sensitive to the fixed parameters conditioning the volume of the insulin compartment or the transfer of insulin between compartments where sensitivities range between 0.18 and 0.48 in response to an increase of $+20 \%$ in the initial values of $V_{1 I}, V_{2 I}, V_{3 I}, K I_{03}$ and $K I_{02}$. The initial value changes of the other fixed parameters $G U N D, K G U G, K G U I, I S N G D$ have only a slight effect on the integrated functions $\left(I S_{T}, I C L_{T}, G U_{T}\right)$. In addition, $I S_{T}$ and $I C L_{T}$ for the glucose tolerance test are also sensitive to $G U M$, which is the parameter conditioning the maximum of glucose utilization (showing sensitivities of $-0 \cdot 30$ for both $I S_{T}$ and $\left.I C L_{T}\right)$. The parameter $I S_{T}$ for the insulin tolerance test is sensitive to $K I_{02}$ and $G E R 0$ (sensitivities of 0.48 and 0.3 respectively). The quantities of glucose utilized $\left(G U_{T}\right)$ are very sensitive to the constant selected for the glucose entry rate function GER0 (sensitivities of 0.71 in the glucose tolerance test and of 0.92 in the insulin tolerance test). This observation is consistent with the fact that the quantities of glucose entering $\left(G E_{T}\right)$ and utilized $\left(G U_{T}\right)$ reach equilibrium over the duration of the tolerance tests.

\section{Results of external validation}

Using similar initial parameters, the model leads to a good simulation (Table 3) of the data of Sechen et al. (1989) and Chilliard \& Ottou (1995). The percentage of variation explained and the standard error of estimates obtained for insulin concentration are comparable with those obtained from the two sets of mean data in the duodenal glucose 
infusion experiment (99.4 and 96.9\% and between 0.017 and $0.04 \mathrm{nmol} / 1$ respectively, for Sechen et al. 1989 and for Chilliard \& Ottou, 1995). However, the percentage of variation for the simulation of glucose concentrations were slightly lower (93.3 and $97.2 \%$ respectively) and the standard error of the estimates $(0.37$ and $0.40 \mathrm{mmol} / \mathrm{l}$ respectively) remained much higher.

\section{Discussion}

This modelling approach combines the information resulting from two distinct tests, which is not possible with the usual methods of data treatment. We estimated six parameters common to both tests, using a simulation carried out on all of the points on the insulin and glucose concentration curves.

\section{Scientific basis of the model}

The present model includes the major crucial processes taking part in the regulation of glucose homeostasis, in particular the secretion and clearance of insulin as well as the utilization of glucose. The sigmoidal form of the insulin secretion function (ISR) is consistent with experimental measurements and other models (Ashcroft, 1981; Sturis et al. 1991). Several equations have been used to simulate sigmoidal functions in glucose metabolism models. In the present model, we decided to use a 'logistic' equation because this parameterization had good estimation proper-

(a)

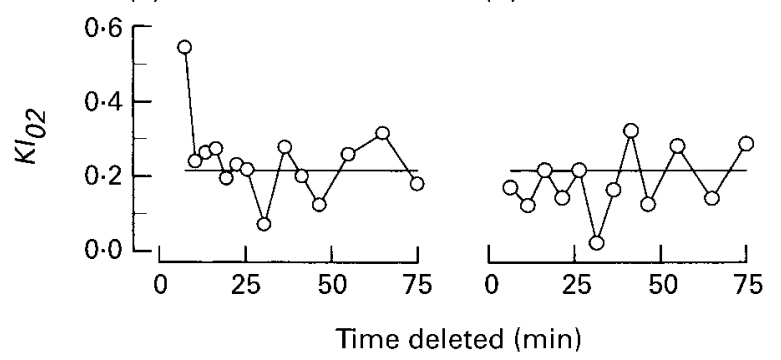

(c)

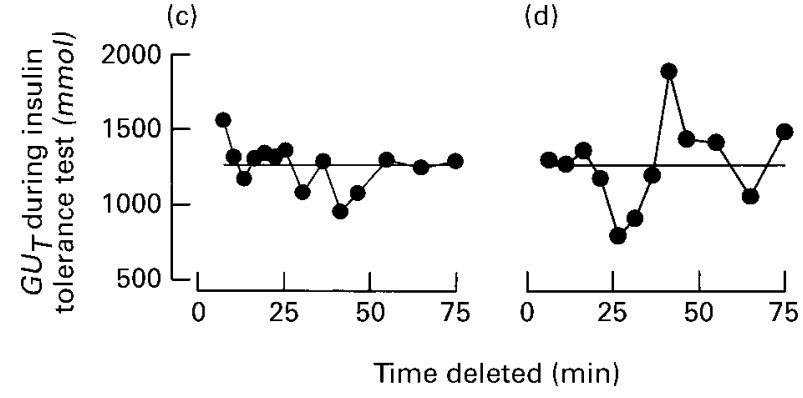

Fig. 4. Examples of the influence of sampling time in determining both parameter values $\left(K I_{02}\right)$ and integrated functions $\left(G U_{T}\right)$ using the Jackknife statistical method. (a), (c), Glucose tolerance test; (b), (d), insulin tolerance test. For details of the model and procedures, see pp. 360-363. Each plot represents the result of a simulation carried out after deleting the plasma insulin and glucose data corresponding to the $n$th time. $K I_{02}$ is the parameter of insulin fractional disappearance rates in interstitial fluid compartment; $G U_{T}$ is the total amount of glucose utilized during the glucose tolerance test. ties (Ratkowsky, 1989). Such equations have already been used in several glucose metabolism models (for example, see Sturis et al. 1991) and this function is frequently used to describe hormonal competition in radioimmunoassay. To describe a sigmoidal function fully, we need an estimation based on four parameters whatever the function used. Since the present data included only plasma insulin and glucose concentrations, we did not have sufficient information to estimate the four parameters and to locate the inflection point precisely. The use of a logistic function imposes symmetry around the inflection point. In future, it may become less appropriate to use a logistic equation to describe more physiologically the insulin secretion function if data other than plasma insulin and glucose concentrations are measured. Nevertheless, it remains difficult to know whether the insulin secretion rate function takes on realistic values because the variation of portal insulin secretion with increasing amounts of injected glucose has not been measured in ruminants. However, values of insulin secretion obtained with the model ranged from basal values to peak values of portal secretion measured in sheep receiving a glucose tolerance test (McCann et al. 1989). The threshold of insulin secretion was estimated at a lower plasma glucose concentration $(1.9 \mathrm{mmol} / \mathrm{l})$ than the levels observed in vitro (Ashcroft, 1981). This also applies to the glucose concentrations corresponding to the point of inflection $(6.6 \mathrm{mmol} / \mathrm{l})$ and the maximum secretion rate $(10-14 \mathrm{mmol} / \mathrm{l})$. This shift of sensitivity towards glucose on the model insulin secretion curve may be explained by the difference of basal glucose concentration between ruminant animals (mean value $3.6 \mathrm{mmol} / \mathrm{l}$ in the studied dairy cows) and single-stomached mammals ( $5 \mathrm{mmol} / \mathrm{l})$.

The cleared insulin parameter values in the model are in agreement with the literature on ruminants (McCann et al. 1989). The hepatic extraction of de novo secreted insulin ranged between 40 and $66 \%\left(K I_{03} 0 \cdot 56\right.$, based on average of data in Tables 1 and 3$)$. The mean value for $K I_{02}(0.085)$ is comparable with the peripheral insulin degradation $(9 \%)$ estimated in human models (Castillo et al. 1994).

The glucose utilization rate function $(G U R)$ is in accordance with several models on single-stomached mammals which use an exponential function to describe non-insulin-dependent glucose utilization and a sigmoidal function to describe insulin-dependent glucose utilization with both functions indexed on glucose levels. In the present model, insulin-dependent glucose utilization increases exponentially with glucose level, as shown by De Fronzo \& Ferrannini (1982), while in most single-stomached mammal models it generally increases linearly with glucose within physiological concentrations (Sturis et al. 1991). However, there is insufficient data on ruminants to define the response of insulin-dependent utilization when glycaemia is increased greatly above basal levels, as seen with glucose tolerance tests, which do not represent a normal physiological concentration for ruminants. With the value adopted for $K G U G(1 \cdot 8)$, the GUR function slows down the utilization of glucose under hypoglycaemic conditions, reaching a maximum as soon as glucose becomes higher than the physiological level (basal level for a ruminant). Nevertheless, the estimates of the non-insulin dependent utilization $\left(G U N D \times\left(1-e^{-K G U G}\right)\right)$, the basal utilization 
Table 3. External validation: results of simulations performed on literature data* $†$

\begin{tabular}{|c|c|c|c|c|c|c|}
\hline & \multicolumn{4}{|c|}{ Chilliard \& Ottou (1995)‡ } & \multicolumn{2}{|c|}{ Sechen et al. (1989)§ } \\
\hline & Control & RO & NA & $\mathrm{NA}+\mathrm{RO}$ & Control & bST \\
\hline ISM (nmol/min) & $7 \cdot 39$ & $5 \cdot 35$ & $7 \cdot 29$ & $7 \cdot 76$ & $14 \cdot 6$ & $15 \cdot 9$ \\
\hline KIS & 0.40 & 0.51 & 0.46 & 0.40 & 0.56 & 0.59 \\
\hline$K I_{02}\left(\min ^{-1}\right)$ & 0.074 & 0.060 & 0.037 & 0.041 & 0.095 & $0 \cdot 121$ \\
\hline$K I_{03}\left(\min ^{-1}\right)$ & 0.58 & 0.51 & 0.74 & 0.53 & 0.72 & 0.62 \\
\hline GERO (mmol/min) & $14 \cdot 7$ & $13 \cdot 8$ & $18 \cdot 5$ & $20 \cdot 5$ & $22 \cdot 2$ & $26 \cdot 5$ \\
\hline GUM $(\mathrm{mmol} / \mathrm{min})$ & $25 \cdot 0$ & $21 \cdot 1$ & $22 \cdot 0$ & $25 \cdot 6$ & 29.5 & $33 \cdot 2$ \\
\hline \multicolumn{7}{|l|}{ Glucose tolerance test } \\
\hline Glucose injected (mmol) & 878 & 878 & 878 & 878 & 813 & 813 \\
\hline$I S_{T}(\mathrm{nmol})$ & 247 & 188 & 349 & 243 & 262 & 287 \\
\hline$I C L_{T}(\mathrm{nmol})$ & 247 & 188 & 350 & 243 & 262 & 287 \\
\hline$G E_{T}(\mathrm{mmol})$ & 2270 & 2138 & 2868 & 3170 & 2775 & 3307 \\
\hline$G U_{T}(\mathrm{mmol})$ & 3154 & 3028 & 3764 & 4056 & 3590 & 4120 \\
\hline \multicolumn{7}{|l|}{ Insulin tolerance test } \\
\hline Insulin injected (nmol) & 576 & 576 & 576 & 576 & 102 & 102 \\
\hline$I S_{T}(\mathrm{nmol})$ & 107 & 70 & 131 & 101 & 65 & 68 \\
\hline$I C L_{T}(\mathrm{nmol})$ & 684 & 647 & 708 & 678 & 168 & 170 \\
\hline$G E_{T}(\mathrm{mmol})$ & 2270 & 2138 & 2868 & 3170 & 2775 & 3307 \\
\hline$G U_{T}(\mathrm{mmol})$ & 2278 & 2162 & 2876 & 3183 & 2775 & 3307 \\
\hline
\end{tabular}

$\mathrm{RO}$, rapeseed oil; NA, niacin; bST, bovine somatotropin; ISM, maximal glucose-stimulated insulin secretion; KIS, glucose sensitivity parameter of insulin secretion rate function; $K I_{02}$ and $K I_{03}$, insulin fractional disappearance rates for compartments 2 and 3; GERO, glucose entry rate function; GUM, parameter allowing maximal insulin-stimulated glucose utilization under euglycaemic conditions; $I S_{T}$, total amount of insulin secreted during a tolerance test; $I C L_{T}$, total amount of insulin cleared; $G E_{T}$, total amount of glucose entering; $G U_{T}$, total amount of glucose utilized.

* Each dataset corresponds to the means for an experimental treatment.

†For details of procedures, see pp. 360-362.

$\ddagger$ Dairy cows received four treatments, i.e. duodenal infusion of water (control) or duodenal infusion of RO or duodenal infusion of NA or a combination of duodenal infusion of NA + RO.

$\S$ Dairy cows were treated with injections of the vehicle (control) or bST.

(equivalent to GER0) and the maximal utilization $(G U N D+G U M)$ have the same orders of magnitude as the values obtained by Debras et al. (1989) in goats in mid-lactation under euglycaemic hyperinsulinaemic clamps (per kg BW: GUND $\times\left(1-e^{-K G U G}\right) 14.8 v$. $20.6 \mu \mathrm{mol} / \mathrm{min}$, GERO 29.7 v. $23.6 \mu \mathrm{mol} / \mathrm{min}, G U M+$ GUND $54 \cdot 1$ v. $40.6 \mu \mathrm{mol} / \mathrm{min})$.

To limit the complexity of the model, the glucose entry rate function was assumed to be constant, which may appear an oversimplification. The model was initially tested with a glucose entry dependent on glucose concentration according to a sigmoidal curve consistent with the model proposed by Cobelli et al. (1982). However, during the entire glucose tolerance test, minimal values of the glucose entry rate were observed and they increased only very slightly $(20 \%)$ at the end of the insulin tolerance test. This is consistent with observations in dairy goats under euglycaemic clamps, in which the glucose entry rate becomes minimal as soon as the plasma insulin concentration exceeds $0 \cdot 35 \mathrm{nmol} / 1$ (Debras et al.1989). For this reason, the model was simplified by adopting a constant glucose entry rate function GERO.

\section{Simulations performed}

The main contribution of the model is to combine the use of two different types of tolerance test in order to improve the estimation of the six parameters, since the simulations carried out using both tests decrease the asymptotic standard errors of estimates compared with simulations carried out using only one of the two tests. The insulin tolerance test improves the estimation of the portal hepatic insulin clearance and glucose entry parameters, while the glucose tolerance test improves the estimation of the insulin secretion parameters. In addition, the standard errors of non-biased parameters estimated using the Jackknife method are relatively low.

To force the model to detect differences between key regulatory functions of glucose homeostasis, we decided to fix all the parameters used to estimate the size of the compartments and the transfer rates between compartments. A sensitivity analysis confirmed that this choice was important because both the insulin secretion and clearance functions are very sensitive to these parameters.

With the six estimated parameters, it would appear possible to detect some modifications of the key functions of glucose homeostasis. For example, significant differences among treatments were observed in the estimation of glucose entry rate $(G E R 0)$, maximum insulin-stimulated glucose uptake $(G U M)$ and, the hepatic insulin clearance parameter $\left(K I_{03}\right)$. Such differences could not be detected using a classical analysis of tolerance tests (Lemosquet $e t$ al. 1997). However, an estimation based on twelve parameters would have been required to define all the modifications of the glucose homeostasis functions in the model. We estimated only two parameters, GUM and GER0, to describe all the modifications of glucose entry and utilization. This may explain why, with increased quantities of injected insulin or glucose, the standard error of estimates of glucose concentration were higher during the tests of Sechen et al. (1989) and Chilliard \& Ottou (1995). In future, to compare individuals in extremely different physiological situations 
(stage of lactation, overnutrition and undernutrition, etc.) which strongly modify all the parameters of glucose homeostasis, it will be necessary to increase the number of estimated parameters. For example, in goats in early lactation under euglycaemic hyperinsulinaemic clamps, the resistance of the body tissues to insulin either resulted in a significant reduction in the maximum insulin-stimulated glucose utilization parameter equivalent to $G U M$ (Debras et al. 1989) or to a significant modification of the sensitivity parameter equivalent to KGUI (Faulkner \& Pollock, 1990; Metcalf \& Weekes, 1990). Nevertheless, to increase the number of parameters estimated without increasing the number of highly correlated parameters, additional assays would be required (e.g. C peptide concentration to calculate insulin secretion, or glucose tracer infusion to calculate turnover).

The model does not accurately describe the glucose and insulin concentrations, either when the glucose concentration is minimal during the insulin tolerance test or just after glucose injection during the glucose tolerance test. In addition, the Jackknife method showed that the estimation of parameters (ISM, KIS, KI $I_{02}, K I_{03}, G E R 0$ and $\left.G U M\right)$ is strongly dependent at both of these stages (Fig. 4). In future, it may be possible to improve the estimation of the parameters through a higher sampling frequency when the glucose concentration is minimal during the insulin tolerance test. Increasing the sampling frequency immediately after the glucose injection during glucose tolerance tests would not necessarily improve the estimation of parameters, since the model does not describe mixing and diffusion processes in the compartments (Shipley \& Clark, 1972). However, modelling this diffusion process would be very complex. To limit this problem, the concentrations of glucose and insulin at the first sampling time $(t<5 \mathrm{~min})$ were not taken into account in the model. Despite this precaution, the values taken by the six estimated parameters were very dependent (Fig. 4) on the first adopted sampling time $(7.5 \mathrm{~min})$. The invalid assumption of instantaneous mixing in each compartment may also explain why a higher $V_{2 G}$ was required to fit the data of Sechen et al. (1989) and Chilliard \& Ottou (1995), corresponding to higher quantities of injected glucose.

Despite all these limitations, the model provides an estimation of the amounts secreted or utilized during the tolerance tests. It also yields low standard errors for these quantities. Unfortunately, it is not easy to compare these quantities with experimental measurements because it remains very expensive to carry out repeated measurement of portal insulin flux or glucose disappearance rate in dairy cows.

\section{Conclusion}

This model, based on the simultaneous interpretation of glucose tolerance and insulin tolerance tests, offers an original alternative for analysing the modifications of glucose homeostasis in dairy ruminants following changes of nutritional or physiological factors without resorting to costly experimental techniques. Using six estimated parameters, the model calculates relative values for the quantities of insulin that are secreted and cleared, or glucose produced and utilized during tolerance tests. In future, it would be interesting to compare these quantities with measured values and to validate the model in a wider range of physiological situations.

\section{Acknowledgements}

The authors are very grateful to Dr G.A. Gagliostro (INTA EEA Balcarce, Argentina) for providing personal data to develop the model and for fruitful discussion, to Dr Y. Chilliard (INRA, Clermont Ferrand-Theix, 63122 Saint Genès-Champanelle, France) for his comments on the modelling of his published data and to John Kennedy for guidance in the writing of this manuscript. M.S.N. Carpenter, 5, rue De Champaubert 35510 CessonSévigné, France, who translated an early draft of this paper, also checked the revised versions.

\section{References}

Annison EF, Bickerstaffe R \& Linzell JL (1974) Glucose and fatty acid metabolism in cows producing milk of low fat content. Journal of Agricultural Science, Cambridge 82, 87-95.

Ashcroft SJH (1981) Metabolic controls of insulin secretion. In The Islets of Langerhans, Biochemistry, Physiology and Pathology, pp. 117-148 [SJ Cooperstein and D Watkins, editors]. Oxford: Academic Press, Inc.

Bergman EN, Reulein SS \& Corlett RE (1989) Effects of obesity on insulin sensitivity and responsiveness in sheep. American Journal of Physiology 257, E772-E781.

Campra JL \& Reynolds TB (1988) The hepatic circulation. In The Liver Biology and Pathbiology, 2nd ed., pp. 911-930 [IM Arias, WB Jakoby, H Popper, D Schachter and A Shafritz, editors]. New York, NY: Raven Press Ltd.

Castillo MJ, Scheen AJ, Letiexhe MR \& Lefèbvre PJ (1994) How to measure insulin clearance. Diabetes/Metabolism Reviews 10, $119-150$

Chilliard Y \& Ottou JF (1995) Duodenal infusion of oil in midlactation cows. 7. Interaction with niacin on responses to glucose, insulin, and $\beta$-agonist challenges. Journal of Dairy Science 78, 2452-2463.

Cobelli C, Federspil G, Pacini G, Salvan A \& Scandellari C (1982) An integrated mathematical model of the dynamics of blood glucose and its hormonal control. Mathematical Biosciences 58, 27-60.

De Fronzo RA \& Ferrannini E (1982) Influence of plasma glucose and insulin concentration on plasma glucose clearance in man. Diabetes 31, 683-688.

Debras E, Grizard J, Aina E, Tesseraud S, Champredon C \& Arnal M (1989) Insulin sensitivity and responsiveness during lactation and dry period in goats. American Journal of Physiology 256, E295-E302.

Faulkner A \& Pollock H (1990) Metabolic responses to euglycaemic hyperinsulinaemia in lactating and non-lactating sheep in vivo. Journal of Endocrinology 124, 59-66.

Ferrannini E \& Cobelli C (1987) The kinetics of insulin in man. I. General aspects. Diabetes/Metabolism Reviews 3, 335-363.

Hocquette JF, Balage M \& Feué P (1996) Facilitate glucose transporters in ruminants. Proceedings of the Nutrition Society $\mathbf{5 5}, 221-236$.

Hovorka R \& Jones RH (1994) How to measure insulin secretion. Diabetes/Metabolism Reviews 10, 91-117.

Hovorka R, Powrie JK, Smith GD, Sönken PH, Carson ER \& Jones RH (1993) Five-compartment model of insulin kinetics and its 
use to investigate action of chloroquine in NIDDM. American Journal of Physiology 265, E162-E175.

Lemosquet S, Rideau N, Rulquin H, Faverdin P, Simon J \& Vérité $R$ (1997) Effect of a duodenal glucose infusion on the relationship of plasma concentrations of glucose and insulin in dairy cows. Journal of Dairy Science 80, 2854-2865.

McCann JP, Bergman EN \& Reimers TJ (1989) Effect of obesity and ovarian steroids on insulin secretion and removal in sheep. American Journal of Physiology 19, E116-E128.

Metcalf JA \& Weekes TEC (1990) Effect of plan nutrition on insulin sensitivity during lactation in the ewe. Journal of Dairy Research 57, 465-478.

Pacini G \& Bergman RN (1986) Minmod: a computer program to calculate insulin sensitivity and pancreatic responsivity from the frequently sampled intravenous glucose tolerance. Computer Methods and Programs in Biomedicine 23, 113-122.

Pfeffer VE, Helfferich B, Bertzbach J \& Lenkeit W (1965) Further studies on the influence of low sodium diet on metabolism of milk cows. 3. Effect on plasma volume and chloride metabolism. Zeitschrift fuer Tierphysiologie Tierernaehrung and Futtermittelkunde 20, 362-364.
Ratkowsky DA (1989) Handbook of Nonlinear Regression Models. New York, NY: Marcel Dekker, Inc.

Sechen SJ, McCutcheon SN \& Bauman DE (1989) Response to metabolic challenges in early lactation dairy cows during treatment with bovine somatropin. Domestic Animal Endocrinology 6, 141-154.

Shipley RA \& Clark RE (1972) Tracer Methods for in vivo Kinetics, London: Academic Press.

Simonoff JS \& Tsai ZL (1986) Jackknife-based estimators and confidence regions in non linear regression. Technometrics $\mathbf{2 8}$, $103-112$.

Steiner EC, Rey TD \& McCroskey PS (1990) Simusolv Modelling and Simulation Software, Midland, MI: The Dow Chemical Company.

Sturis J, Polonsky KS, Mosekilde E \& Van Cauter E (1991) Computer model for mechanisms underlying ultradian oscillations of insulin and glucose. American Journal of Physiology 23, E801-E809.

Turner RC, Rudenski AS, Matthews DR, Levy JC, O'Rahilly P \& Hosker JP (1990) Application of structural model of glucoseinsulin relations to assess beta-cell function and insulin sensitivity. Hormone and Metabolic Research 24, Suppl 1, 66-71. 